\title{
Effects of L-Arginine Pre-treatment on Isoproterenol-Induced Changes in Lipid Metabolism during Experimental Myocardial Injury in Rats
}

\author{
Meenakshisundaram SReEPriYA, ${ }^{1}$ Thiruvengadam DevaKI, ${ }^{1, *}$ \\ and Mohammed NAYEEM ${ }^{2}$ \\ ${ }^{1}$ Department of Biochemistry and Molecular Biology, University of Madras, \\ Guindy Campus, Chennai 600025, Tamil Nadu, India \\ ${ }^{2}$ Department of Physiology and Biochemistry, Madras Veterinary College, \\ Vepery, Chennai 600007, Tamil Nadu, India
}

(Received September 14, 1998)

\begin{abstract}
Summary The biochemical effects of L-arginine pre-treatment against isoproterenol-induced changes with respect to lipid metabolism were studied in male albino rats. The levels of cholesterol, triglycerides, and free fatty acids were estimated in both serum, and the myocardial tissue. Levels of lipid peroxides, LDL and HDL cholesterol in the serum, and total lipids and phospholipids in the myocardial tissue were also estimated in the normal and experimental rats. Isoproterenol-induced significant increases in the levels of LDL cholesterol and other lipid components in the serum and myocardial tissue (except phospholipids) were restored to near normal levels in the rats given L-arginine pre-treatment prior to the induction of myocardial stress by isoproterenol administration, thereby exhibiting a protective effect of L-arginine against isoproterenol-induced myocardial damage in rats. Results of our preliminary study thus reveal that L-arginine is cardioprotective in experimental rats.
\end{abstract}

Key Words: L-arginine, cardioprotection, isoproterenol, myocardial damage, LDL cholesterol, lipid metabolism

Isoproterenol is a synthetic catecholamine and beta adrenergic agonist that causes severe stress in the myocardium, resulting in infarct-like necrosis of the heart muscle [1]. Isoproterenol-induced myocardial necrosis showed membrane permeability alterations which bring about the loss of function and integrity of myocardial membranes [2]. During myocardial infarction induced by isoproterenol, there is an increase in the level of myocardial lipids [3].

*To whom correspondence should be addressed. 
Isoproterenol is known to generate free radicals and to stimulate lipid peroxidation. The formation of free radicals as well as accumulation of lipid peroxides has thus been recognized as one of the possible biochemical mechanisms for the myocardial damage caused by this catecholamine [1].

L-Arginine is a semi-essential amino acid and stimulates the production of endothelium-derived nitric oxide (NO) [4]. Its rate of endogenous production is slow and insufficient to meet physiological needs; hence it is supplemented in the diet [5]. Recently nitric oxide derived from L-arginine in the endothelium has been proved to possess significant cardioprotective properties. It is a chemical messenger that acts as a potent vasodilator and increases coronary flow [6]. Endogenously derived nitric oxide plays important bioregulatory roles in a number of physiological processes, such as control of blood pressure, neurotransmission, platelet aggregation, and the cytostatic and cytotoxic action of macrophage cells $[7,8]$.

The main biological function of nitric oxide seems to be the stimulation of the soluble guanylate cyclase to elevate cGMP. Increasing cGMP promotes relaxation of vascular smooth muscle and inhibits platelet aggregation as well as platelet adhesion to endothelium [9]. Nitric oxide also inhibits lipoxygenase and cyclo oxygenase activity towards lipid oxidation [10]. Hence the biological activity of L-arginine is manifested through the formation of nitric oxide in the endothelium.

Biochemical studies on the effect of L-arginine against isoproterenol-induced changes with respect to lipid metabolism have not been previously reported. In the present investigation, we examined the efficacy of L-arginine pre-treatment against isoproterenol-induced changes in lipid metabolism during experimental myocardial injury in rats.

\section{MATERIALS AND METHODS}

Adult male Wistar strain albino rats weighing about $140-180 \mathrm{~g}$ were obtained from Fredrick Institute for Plant Protection and Toxicology, Padappai, Chennai. They were acclimatized to animal house conditions, and were fed commercial pellet rat chow (Hindustan Lever Limited, Bangalore, India) and given water ad libitum. Isoproterenol and L-arginine were obtained from Sigma Chemical Company, St. Louis, MO, USA. The animals were divided into four groups (of six animals each) according to the experimental regimens indicated below:

Group I served as the normal control. Group II was rats with myocardial stress induced by two intraperitoneal injections of isoproterenol $(150 \mathrm{mg} / \mathrm{kg}$ body wt./day for 2 days, one on each day). Group III was normal rats given L-arginine pre-treatment for 21 days $(250 \mathrm{mg} / \mathrm{kg}$ body wt./day). Group IV was rats given L-arginine pre-treatment for 21 days prior to the induction of myocardial stress by isoproterenol administration.

After the experimental period, the animals were killed by cervical decapitation. Blood was collected, and the serum separated was used for the estimation of 
cholesterol [11], triglycerides [12], free fatty acids [13, 14] and lipid peroxides [15]. HDL and LDL fractions were separated from serum according to the dual precipitation technique [16]. The heart was dissected out immediately after the sacrifice and washed in ice-cold saline. Myocardial cholesterol, phospholipid [17], triglycerides, and free fatty acids, were estimated after extracting the total lipid by the method of Folch et al. [18]. The values were expressed as the mean \pm SD. Statistical difference was analyzed by Student's $t$-test, and $p$-values were determined.

\section{RESULTS}

Table 1 shows the levels of cholesterol, triglycerides, and free fatty acids in the serum of normal and experimental rats. In the isoproterenol-treated rats (Group II), there was a significant increase in the levels of the lipids. Table 2 shows the levels of HDL and LDL cholesterol and of lipid peroxides in the serum of both normal and experimental rats. Levels of lipid peroxides and LDL cholesterol were significantly increased in the Group II rats, whereas they were significantly decreased in the Group IV animals. The Group III rats did not show any significant changes in the levels of lipids as compared with the values for normal rats. Table 3 shows the levels of total lipids, cholesterol, triglycerides, free fatty acids, and phospholipids in the heart of normal and experimental rats. The levels of the myocardial lipids except phospholipids showed a significant increase in the iso-

Table 1. Levels of cholesterol, triglycerides and free fatty acids in the serum of the four groups of animals.

\begin{tabular}{clll}
\hline \multicolumn{1}{c}{ Groups } & Cholesterol & Triglycerides & Free fatty acids \\
\hline I. Normal & $78.66 \pm 6.01$ & $39.66 \pm 2.59$ & $22.67 \pm 1.06$ \\
II. Isoproterenol & $97.80 \pm 10.12^{* * *}$ & $59.50 \pm 6.21^{* * *}$ & $33.00 \pm 1.98^{* * *}$ \\
III. L-Arginine & $80.33 \pm 7.90^{\mathrm{NS}}$ & $40.50 \pm 5.20^{\mathrm{NS}}$ & $24.00 \pm 3.26^{\mathrm{NS}}$ \\
IV. L-Arginine+Isoproterenol & $86.20 \pm 6.98^{\mathrm{NS}}$ & $44.00 \pm 3.01^{*}$ & $26.17 \pm 2.89^{*}$ \\
\hline
\end{tabular}

Values are expressed as the mean \pm SD for six animals in each group. Units: $\mathrm{mg} / \mathrm{dl}$ of serum. As compared with control (Group I). ${ }^{* * *} p<0.001,{ }^{*} p<0.05$, NSNot significant.

Table 2. Levels of lipid peroxides and of LDL and HDL cholesterol in the serum of the four groups of animals.

\begin{tabular}{|c|c|c|c|}
\hline Groups & Lipid peroxides & LDL cholesterol & HDL cholesterol \\
\hline I. Normal & $3.10 \pm 0.35$ & $43.16 \pm 3.91$ & $21.8 \pm 2.02$ \\
\hline II. Isoproterenol & $5.93 \pm 0.61 * * *$ & $58.50 \pm 5.21^{* * *}$ & $12.0 \pm 1.21^{* * *}$ \\
\hline III. L-Arginine & $3.23 \pm 0.42^{\mathrm{NS}}$ & $43.66 \pm 3.98^{\mathrm{NS}}$ & $21.0 \pm 2.23^{\mathrm{NS}}$ \\
\hline IV. L-Arginine +Isoproterenol & $3.80 \pm 0.59^{*}$ & $49.00 \pm 4.20^{*}$ & $18.5 \pm 1.80^{*}$ \\
\hline
\end{tabular}

Vol. 25, No. 3, 1998 
Table 3. Levels of cholesterol, triglycerides, free fatty acids, phospholipids and total lipid in the heart of the four groups of animals.

\begin{tabular}{llllll}
\hline \multicolumn{1}{c}{ Groups } & Cholesterol & Triglycerides & $\begin{array}{c}\text { Free fatty } \\
\text { acids }\end{array}$ & Phospholipids & Total lipids \\
\hline I. Normal & $5.00 \pm 0.37$ & $4.28 \pm 0.33$ & $0.12 \pm 0.01$ & $27.17 \pm 5.5$ & $52.12 \pm 4.20$ \\
II. Isoproterenol & $8.63 \pm 0.85^{* * *}$ & $7.63 \pm 0.75^{* * *}$ & $0.20 \pm 0.01^{* * *}$ & $17.50 \pm 3.3^{* *}$ & $65.94 \pm 6.15^{* * *}$ \\
III. L-Arginine & $5.20 \pm 0.13^{\mathrm{NS}}$ & $4.74 \pm 0.64^{\mathrm{NS}}$ & $0.13 \pm 0.01^{\mathrm{NS}}$ & $26.50 \pm 2.4^{\mathrm{NS}}$ & $53.22 \pm 5.65^{\mathrm{NS}}$ \\
IV. L-Arginine+ & $6.00 \pm 0.75^{*}$ & $5.01 \pm 0.64^{*}$ & $0.15 \pm 0.02^{*}$ & $21.16 \pm 3.01^{*}$ & $60.16 \pm 5.90^{*}$ \\
$\quad$ Isoproterenol & & & & & \\
\hline
\end{tabular}

Values are expressed as the mean \pm SD for six animals in each group. Units: $\mathrm{mg} / \mathrm{g}$ of wet tissue. As compared with control (Group I). ${ }^{* * *} p<0.001,{ }^{* *} p<0.01,{ }^{*} p<0.05$, NSNot significant.

proterenol-intoxicated rats, which levels were reduced markedly in rats given L-arginine pre-treatment prior to isoproterenol administration. The levels of phospholipids showed a slight decrease in the isoproterenol-administered rats, whereas the levels of phospholipids were maintained near normal in the Group IV rats.

\section{DISCUSSION}

The increase in the levels of serum lipids due to isoproterenol administration is an evidence for its known hyperlipidemic effect [19]. Isoproterenol mainly increased the LDL cholesterol level. LDL carries the bulk of cholesterol in the blood and leads to the buildup of harmful deposits in the arteries, and thus favors coronary heart disease [20]. Hence there is a positive correlation between the risk of developing ischemic heart disease and serum LDL cholesterol [21]. High levels of circulating cholesterol and its accumulation in the heart tissue are well associated with cardiovascular damage [22].

Increased cholesterol levels in the heart during myocardial infarction could be due to increased direct uptake of LDL from the blood by the tissues [23]. Hypertriglyceridemia was observed in our isoproterenol-treated rats, and such a state was also reported in association with cardiovascular disturbances [24]. The levels of triglycerides were much reduced in Group IV rats as compared with those of Group II rats. The free fatty acids liberated by the adipose tissue are taken up by the myocardium, and the activity is proportional to the free fatty acid concentration in the coronary sinus. The heart utilizes free fatty acids for its energy requirements, and the excess free fatty acid is used for the synthesis of glycerides [25]. This accounts for the increased triglyceride levels during isoproterenol administration.

The levels of free fatty acids were found to be increased in the isoproterenoladministered group of rats. The increased peroxidation of the membrane phospholipids releases free fatty acids by the action of phospholipase A2 [26]. $\mathrm{Ca}^{2+}$ ions have been reported to be one of the inducers of phospholipase A2. So the 
increased levels of free fatty acids in the isoproterenol-administered group might be due to the indirect effect of the calcium level, which was reported to be elevated upon isoproterenol-treatment [27]. The endothelial damage induced by isoproterenol causes lipid mobilization, which leads to increased free fatty acids in circulation, which in turn causes platelet aggregation and hastens endothelial damage. The resultant smooth muscle cell proliferation and fat deposition causes atherosclerotic plaques to develop in blood vessels.

Isoproterenol also promotes lipolysis in the myocardium $[28,29]$. Increased lipolysis of depot triglycerides liberates free fatty acids from adipose tissue stores [30-33]. Fatty acid composition of tissue lipids may also play an important role in lipid peroxidation, as fatty acids are important substrates for microsomal lipid peroxidation [34]. The accumulation of lipids in the heart of rats treated with isoproterenol could be responsible for the functional disorders. Elevation of lipid peroxides may be attributed to the accumulation of lipids in heart [35], and increased levels of lipid peroxides may be a causative factor in the irreversible damage to the myocardial membrane [36].

L-Arginine reversed the elevated levels of LDL cholesterol probably by regulating cholesterogenesis and by its inhibitory effect on lipid peroxidation, thereby reducing the levels of lipid components and hence the levels of LDL.

The inhibitory effect of L-arginine on lipid peroxidation is mediated via the nitric oxide pathway. It should be noted that the combination of reactive oxygen species and nitrogen intermediates may be antagonistic in some circumstances. Accordingly, it is interesting to observe that nitric oxide produced from L-arginine actually protects mammalian cells against oxidant injury, perhaps by forming iron nitrosyl complexes (making iron less available for catalysis of pro-oxidant reactions), thereby directly scavenging radical species such as ${ }^{\circ} \mathrm{OH}$. Nitric oxide may also antagonize oxidant membrane injury by terminating lipid peroxidation reactions [37]. Direct reaction between nitric oxide and lipid peroxyl radical forms a lipid nitroso adduct. This radical-radical interaction results in the termination of lipid peroxidation chain reaction and stops further initiation $[38,39]$.

These results have confirmed the efficacy of L-arginine as a potent cardioprotective agent. The results also confirm the protective and inhibitory effect of L-arginine against isoproterenol-induced lipid peroxidation.

\section{REFERENCES}

1. Sushamakumari, S., Jayadeep, A., Sureshkumar, J.S., and Menon, V.P. (1989): Effects of carnitine on malondialdehyde, taurine, glutathione levels in heart of rats subjected to myocardial stress by isoproterenol. Indian J. Exp. Biol., 27, 134-137.

2. Todd, G.L., Cullan, G.E., and Cullan, G.M. (1980): Iso-proterenol-induced myocardial necrosis and membrane permeability alterations in the isolated perfused rabbit heart. Exp. Mol. Pathol., 33, 43-54.

3. Manjula, T.S., and Shyamala Devi, C.S. (1993): Effect of aspirin in isoproterenol induced changes in lipid metabolism in rats. Indian J. Med. Res., 98, 30-33.

Vol. 25, No. 3, 1998 
4. Egashira, K., Hirooka, Y., Kuga, T., Mohri, M., and Takeshita, A. (1996): Effects of Larginine supplementation on endothelium-dependent coronary vasodilation in patients with angina pectoris and normal coronary arteriograms. Circulation, 94, 130-134.

5. West, E.S., Todd, W.R., Mason, H.S., and VanBruggen, J.T. (1996): Aminoacids, in Text Book of Biochemistry, ed. by West, E.S., and Toda, W.R., 4th ed., Oxford and IBH Publishing Company Private Limited, New Delhi, pp. 1143-1263.

6. Zhu, B.Q., Sun, Y.P., Richar, E., Sievers, R.S., Jonathan, L., and Shuman, B.S. (1996): L-Arginine decreases infarct size in rats exposed to environmental tobacco smoke. Am. Heart. J., 132, 91-100,

7. Moncada, S., Palmer, R.M.J., and Higgs, E.A. (1991): Nitric oxide physiology, pathophysiology and pharmacology. Pharmacol. Rev., 43, 109-142.

8. Marletta, M.A. (1989): Nitric oxide: Biosynthesis and biological significance. Trends Biochem. Sci. 14, 488-492.

9. Ignarro, L.J. (1990): Biosynthesis and metabolism of endothelium-derived relaxing nitric oxide. Annu. Rev. Pharmacol. Toxicol., 30, 535-560.

10. Kanner, J., Harel, S., and Granit, R. (1991): Nitric oxide as an antioxidant. Arch. Biochem. Biophys., 289, 130-136.

11. Parekh, A.C., and Jung, D.H. (1970): Cholesterol determination with ferric acetate-uranyl acetate and sulphuric acid-ferrous sulphate reagents. Anal. Chem., 42, 1423-1427.

12. Rice, E.W. (1970): Triglycerides in serum, in Standard Methods in Clinical Chemistry, ed. by Roedrick, P., and McDonald, R.P., Academic Press, New York, p. 215.

13. Hron, W.T., and Menahan, L.A. (1981): A sensitive method for the determination of free fatty acids in plasma. J. Lipid Res., 122, 377-381.

14. Itaya, K. (1977): A more sensitive and stable colorimetric determination of free fatty acids in plasma. J. Lipid Res., 18, 663-665.

15. Yagi, K. (1976): Simple fluorimetric assay for lipid peroxides in blood plasma. Biochem. Med., 15, 212-215.

16. Burstein, M., and Scholnick, H.R. (1972): Precipitation of chylomicron and very low density lipoprotein from human serum with sodium lauryl sulphate. Life Sci., 11, 177-184.

17. Fiske, C.H., and Subbarow, Y. (1925): The colorimetric determination of phosphorus. $J$. Biol. Chem., 66, 375-400.

18. Folch, J., Lees, M., and Stanley, G.H.S. (1957): A simple method for the isolation and purification of total lipids from animal tissues. J. Biol. Chem., 226, 497-509.

19. Wexler, B.C. (1973): Protective effects of propranolol on isoproterenol-induced myocardial infarction in arteriosclerotic rats. Atherosclerosis, 18, 11-17.

20. Goldstein, J.L., and Brown, M.S. (1984): Progress in understanding the LDL receptor and HMG CoA reductase to membrane proteins that regulate the plasma cholesterol. J. Lipid Res., 25, 1450-1460.

21. Manjula, T.S., Geetha, A., Ramesh, T.G., and Shyamala Devi, C.S. (1992): Reversal of changes of myocardial lipids by chronic administration of aspirin in isoproterenol-induced myocardial damage in rats. Indian J. Physiol. Pharmacol., 36, 47-50.

22. Joan, F., Peter, Z., and Philip, D. (1984): Cholesterol, in Clinical Chemistry on Diagnosis and Treatments, ed. by Amold, E., and Lyold, L., PG Publishing, New Delhi, pp. 230-256.

23. Mathew, S., Menon, P.V.G., and Kurup, P.A. (1981): Changes in myocardial and aortic lipids, lipolytic activity and fecal excretion of sterols and bile acids in isoproterenol-induced myocardial infarction in rats. Indian J. Biochem. Biophys., 18, 131-134.

24. Freedman, D.S., Gruchow, H.W., Anderson, A.J., Rimm, A.A., and Barboriak, J.J. (1988): Relation of triglyceride levels to coronary artery disease. The Milwaukee cardio-vascular data registry. Am. J. Epidemiol., 127, 1118-1130.

25. Heindel, J.J., Orci, L., and Jenarenaud, B. (1975): Fat mobilization and its regulation by hormones and drugs in white adipose tissue, in International Encyclopaedia of Pharmacology and Therapeutics. Pharmacology of Lipid Transport and Atherosclerotic Processes, Vol. 1, ed. by Masro, S., Pergamon Press, Oxford, pp. 175-373. 
26. Chein, K.R., Sherman, S.C., Mittamcht, S., Jr., and Faber, J.L. (1980): Microsomal structure and function subsequent to calcium activation of an endogenous phospholipase. Arch. Biochem. Biophys., 205, 614-622.

27. Shen, A.C., and Jennings, R.B. (1972): Myocardial $\mathrm{Ca}^{++}$and $\mathrm{Mg}^{++}$in acute ischaemic injury. Am. J. Pathol., 67, 417-421.

28. Lech, J.J., Jesmok, G.J., and Calvert, D.N. (1977): Effects of drugs and hormones on lipolysis in heart. Fed. Proc., 36, 2000-2008.

29. Severson, D.L. (1979): Regulation of lipid metabolism in adipose tissue and heart. Can. J. Physiol. Pharmacol., 57, 923-937.

30. Kruger, F.A., Leighty, E.G., and Weissler, A.M. (1967): Cathecholamine stimulation of myocardial lipolysis and fatty acid reesterification. J. Clin. Invest., 46, 1080-1081.

31. Fain, J.N., and Shepherd, R.E. (1975): Free fatty acids as regulators of adenylatecyclase and cyclic-3'-5'AMP accumulation in fat cells. J. Biol. Chem., 250, 6586-6592.

32. Steinberg, D. (1976): Interconvertible enzymes in adipose tissue regulated by cyclic AMP-dependent protein kinase. Adv. Cyclic Nucleotide Res., 7, 157-198.

33. Steinberg, D., and Khoo, J.C. (1977): Hormone sensitive lipase of adipose tissue. Fed. Proc., 36, 1986-1990.

34. Chein, K.R., Abrams, J., Serroni, A., Martin, J.T., and Farber, J.L. (1978): Accelerated phospholipid degradation and associated membrane dysfunction in irreversible, ischaemic liver cell injury. J. Biol. Chem., 253, 4809-4817.

35. Mead, J.F. (1976): Free radical mechanisms of lipid damage and consequences for cellular membranes, in Free Radicals in Biology, ed. by Pryor, W.A., Academic Press, New York, p. 51 .

36. Tappel, A.I. (1980): Measurement of and protection from in vivo lipid peroxidation, in Free Radicals in Biology, ed. by Pryor, W.A., Academic Press, New York, pp. 1-47.

37. Fang, F.C. (1997): Mechanisms of nitric-oxide related antimicrobial activity. J. Clin. Invest., 99, 2818-2825.

38. Goss, S.P.A., Hogg, N., and Kalyanaraman, B. (1995): The antioxidant effect of spermine NONOate in human low-density lipoprotein. Chem. Res. Toxicol., 8, 800-806.

39. Rubbo, H., Radi, R., Trujillo, M., Telleri, R., Kalyanaraman, B., Barnes, S., Kirk, M., and Freeman, B.A. (1994): Nitric oxide regulation of superoxide and peroxynitrite-dependent lipid peroxidation. Formation of novel nitrogen-containing lipid derivatives. J. Biol. Chem., 269, 26066-26075. 